\title{
COUPLED MACRO-MICRO MODELLING OF THE SECONDARY MELTING OF TURBINE DISC SUPERALLOYS
}

\author{
P.D. Lee, R. Lothian, L.J. Hobbs, and M. McLean \\ Department of Materials, Imperial College \\ London, UK
}

\begin{abstract}
$\underline{\text { Abstract }}$
A model of the macroscopic fluid flow, heat transfer and electromagnetic effects during secondary melting was developed and coupled to microstructural feature prediction. The importance of the different driving forces on the macroscopic model was explored using the test cases of vacuum arc remelting (VAR) and electron beam button melting (EBBM) of IN718. The predictions of the surface flows and final microstructure in the EBBM process were compared to experimental results.
\end{abstract}

\section{Introduction}

The demands of the aerospace industry have led to the development of a range of alloys with specific properties to satisfy the design requirements for different parts of the gas turbine engine. For turbine blades, the need is for a balance of very high temperature (up to $-1100^{\circ} \mathrm{C}$ ) creep strength and corrosion resistance, whilst turbine discs have lower operating temperatures $\left(\sim 800^{\circ} \mathrm{C}\right)$. However, the demands for increasing power, through higher turbine speeds with associated increased centrifugal forces, and reduced engine weight have required significant increases in stresses carried by the discs. The development of alloys for turbine disc applications has concentrated on increasing the yield strength of the materials bringing a concomitant decrease in toughness, which has made the fracture characteristics of these materials very sensitive to the presence of defects. Consequently, it is now standard practice to improve the quality of turbine disc alloys, initially produced by vacuum induction melting (VIM), by subjecting them to one or more secondary melting processes. These processes include vacuum arc re-melting (VAR), electroslag re-melting (ESR) and electron beam cold hearth refining (EBCHR). The quality improvements obtained are a finer microstructure, less macrosegregation and a reduced inclusion content.

In the past, the design and optimisation of secondary melting processes for new alloys has involved a great deal of expensive trial and error. Numerical modelling can, in principle, provide a less expensive route to reliable and efficient alloy processing. This paper evaluates the viability of combining macroscopic process models with empirical and theoretical treatments of microstructure formation for the accurate prediction of the ingot microstructure and defect susceptibility.
Theory

\section{Macro-Model}

There are many factors involved in the VAR process. Correspondingly, a detailed description of the real installation being modelled and accurate thermophysical data for the alloy involved must be supplied to any model which aspires to give accurate predictions. Factors to be dealt with include: heat transfer, fluid flow, magnetohydrodynamics (including both the Lorentz force and Joule heating), and solidification.

The modelling of the heat transfer, fluid flow, and solidification was performed using the commercial finite volume code Fluenit with subroutines added to handle the spatially and temporally varying heat flux and surface tension gradient boundary conditions. The calculation of the Lorentz force and Joule heating was also coded in, but proved an insignificant effect in EBBM, although it is crucial in VAR. The equations being solved are listed below.

\section{Energy Equation}

The energy equation to be solved is:

$$
\frac{\partial}{\partial t}(\rho h)+\nabla \cdot(\rho \mathbf{u} h-k \nabla T-\tau \cdot \mathbf{u})=\frac{\partial p}{\partial t}+\mathbf{u} \cdot \nabla p+S_{h},
$$

where $h$ is the static enthalpy, $p$ is the static fluid pressure, $\rho$ is the density, $k$ is the thermal conductivity, $t$ is time, $\mathbf{u}$ is the fluid velocity, $T$ is temperature and $\tau$ is the viscous stress tensor. $S_{h}$ represents heat sources, including Joule heating (VAR), electron beam heating (EBBM), energy losses by radiation and conduction and latent heat evolution. The last of course, plays a crucial role in solidification modelling.

\section{Momentum and Continuity Equations}

The velocity of liquid, $\mathbf{u}$, is given by the momentum equation ${ }^{1}$ :

$$
\frac{\partial}{\partial t}(\rho \mathbf{u})+\nabla \cdot(\rho \mathbf{u} \otimes \mathbf{u}+\mathrm{p} \mathbf{I}-\tau)=\rho \mathbf{g}+\mathbf{F},
$$

where $\mathbf{I}$ is the $3 \times 3$ identity tensor, $\mathbf{g}$ is the gravitational acceleration, and $\mathbf{F}$ is the sum of all other body forces (e.g. the Marangoni force or the Lorentz force).

The stress tensor $\tau$ has components:

$$
\tau_{i j}=\left[\mu\left(\frac{\partial u_{i}}{\partial x_{j}}+\frac{\partial u_{i}}{\partial x_{i}}\right)\right]-\frac{2}{3} \mu \frac{\partial u_{l}}{\partial x_{l}} \delta_{i j}
$$

where $\mu$ is the viscosity. 
To solve for the momentum and pressure, the mass conservation equation must also be satisfied:

$$
\frac{\partial \rho}{\partial t}+\nabla \cdot(\rho \mathbf{u})=S_{m}
$$

$S_{m}$ is the mass added to the continuous phase from any dispersed phase or as a source (e.g. due to droplets from the consumable electrode). In the present work, $S_{m}$ has been taken to be zero, since filling is not modelled.

\section{Electromagnetic Effects}

The magnetic induction $\mathbf{B}$ in a magnetised fluid is calculated from the induction equation:

$$
\frac{\partial \mathbf{B}}{\partial t}=\nabla \times(\mathbf{u} \times \mathbf{B})+\eta \nabla^{2} \mathbf{B}
$$

Here $\eta=\left(\mu_{e} \sigma\right)^{-1}$ is the magnetic diffusivity, where $\mu_{\mathrm{e}}$ is the magnetic permeability (assumed in this work to take its vacuum value $\mu_{0}$ ) and $\sigma$ is the conductivity of the fluid. In the steady state, the LHS of (5) is zero. Furthermore, we may estimate the relative magnitudes of the terms on the RHS. The ratio of the first term, representing advection of field by the flow, to the second, representing field diffusion through Joule dissipation is given by the magnetic Reynolds number:

$$
\mathrm{R}_{\mathrm{m}}=\frac{\mathrm{Lv}}{\eta} \text {. }
$$

The length scale $\mathrm{L}$ is typically $1 \mathrm{~m}$ in industrial scale remelting processes and $\eta \approx 1 \mathrm{~m}^{2} / \mathrm{s}$, so the diffusion term dominates if the typical flow velocity $v$ is much less than $1 \mathrm{~m} / \mathrm{s} . R_{m}$ is correspondingly smaller on the smaller scales of pilot VAR and EBBM melts. It has been normal to neglect the advection term in such studies and this approach is justified a posteriori if computed velocities are indeed small. With these assumptions, B decouples from the fluid flow and may be calculated from the equation:

$$
\nabla^{2} \mathbf{B}=0 .
$$

This equation was solved subject to the imposition of normal current components on the boundaries of the ingot.

The current density, $\mathbf{J}$, Lorentz Force, $\mathbf{F}_{\mathrm{e}}$, and Joule heating, $\mathbf{Q}_{\text {joule }}$, werc calculated from the equations:

$$
\begin{gathered}
\mu_{0} \mathbf{J}=\nabla \times \mathbf{B} \\
\mathbf{F}_{\mathrm{e}}=\mathbf{J} \times \mathbf{B}
\end{gathered}
$$

and

$$
\mathrm{Q}_{\text {joule }}=\frac{\mathbf{J} \cdot \mathbf{J}}{\sigma} .
$$

In practice, a 2-D axisymmetric model, which considerably reduces computing time, was adopted.

\section{Micro-Models}

As summarised by McLean ${ }^{2}$ the primary dendrite arm spacing, $\lambda_{1}$, in superalloys has been shown by many authors to be a function of the thermal gradient and the growth velocity both experimentally and theoretically. Recently Lu and Hunt ${ }^{3}$ developed a numerical model of cellular and dendritic growth to predict the cell and dendrite spacing as well as the undercooling at the tip. Hunt and $\mathrm{Lu}^{4}$ provided an analytic expression fitted to their numerical model results that predicts the minimum stable half-spacing of dendrites.
Cast in terms of non-dimensional parameters", Hunt and $\mathrm{Lu}$ found for the case of $G^{\prime}>1 \times 10^{-10}$ and $0.068<k<0.69$ that the dimensionless dendrite half spacing, $\lambda^{\prime}$, is given by:

$$
\lambda^{\prime}=0.7798 \times 10^{-1} V^{(a-0.75)}\left(V^{\prime}-G^{\prime}\right)^{0.75} G^{-0.6028},
$$

where:

$$
a=-1.131-0.1555 \log _{10}(G)-0.7589 \times 10^{-2}\left[\log _{10}\left(G^{\prime}\right)\right]^{2} .
$$

To determine $\lambda_{1}$ the thermal gradient and front velocity were calculated using the macro-model. As a cell passes through the liquidus temperature the nearest neighbours' temperatures were used to calculate the thermal gradient normal to the isotherm. Hunt and $L u$ specify $G$ as the solid thermal gradient, referring to the dendrite tip as the solid. In the macro model the mushy zone is treated as a continuum, making the thermal gradient close to the liquidus temperature an appropriate approximation for $G$.

The value of $V$ used to determine $\lambda_{1}$ was the velocity of the liquidus isotherm, $V_{\text {liq. }}$. This velocity was determined using a central difference approximation of the following derivative:

$$
V_{l i q}=\left.\frac{\partial n}{\partial t}\right|_{T=T_{l i q}}=\frac{1}{\left.\frac{\partial t}{\partial n}\right|_{T=T_{l i q}}}=\frac{1}{\frac{\partial t_{l}}{\partial n}},
$$

where $t_{l}$ is the time at which the local temperature attains the liquidus value and $n$ is the direction normal to the liquidus isotherm.

The ripening or local solidification time $t_{s}$, is defined as the time for which the dendrite is in the mushy zone. The secondary dendrite arm spacing, $\lambda_{2}$, is related to $t_{s}$ by:

$$
\lambda_{2}=-7.0+12.5 t_{s}^{0.33}[\mu \mathrm{m}] \text {. }
$$

The carbide size, $\lambda_{\text {carb }}$, has also been shown experimentally to be a function of the local solidification time by Jardy et al. ${ }^{5}$, who suggests the following formula for IN718:

$$
\lambda_{c a r b}=\sqrt[3]{80.9+9.16 t_{s}}[\mu \mathrm{m}]
$$

\section{Comparison of Different Driving Forces}

In order to estimate the relative importance of Lorentz, Marangoni and buoyancy forces, the relevant dimensionless numbers are constructed using the typical magnitudes listed in Table I.

To compare the relative importance of Marangoni and buoyancy forces, we evaluate the dimensionless quantity:

$$
M \equiv \frac{\frac{\partial \gamma}{\partial T}}{\frac{\partial \rho}{\partial T} L^{2} g} .
$$

\footnotetext{
* The non-dimensional parameters are $G^{\prime}=\frac{G \Gamma k}{\Delta T_{o}^{2}}, V^{\prime}=\frac{V \Gamma k}{D \Delta T_{o}}$ and $\lambda^{\prime}=\frac{\lambda \Delta T_{0}}{\Gamma k}$, where $\wedge T_{o}$ is the undercooling for a planar front given by $\Delta T_{o}=\frac{m C_{o}(k-1)}{k} . G, V$, $\Gamma, D, m, C_{o}$, and $k$ are respectively the solid temperature gradient, velocity, Gibbs-Thomson coefficient, liquid diffusion coefficient, liquidus slope, bulk composition, and distribution coefficient ${ }^{4}$.
} 
Table I. Nominal properties used to calculate the relative importance of the driving forces during EBBM and VAR of nickel based superalloys.

\begin{tabular}{c|cccc}
\hline Property & Symbol & EBBM & VAR & Units \\
\hline Density & $\rho$ & 8000 & 8000 & $\mathrm{~kg} / \mathrm{m}^{3}$ \\
Characteristic length & $\mathrm{L}$ & 0.01 & 0.1 & $\mathrm{~m}$ \\
Surface tension & $\partial \gamma / \partial T$ & $-1 \times 10^{-4}$ & $-1 \times 10^{-4}$ & $\mathrm{~N} / \mathrm{m} / \mathrm{K}$ \\
gradient & & & & \\
Density gradient & $\partial \rho / \partial T$ & 0.1 & 0.1 & $\mathrm{~kg} / \mathrm{m}^{3} / \mathrm{K}$ \\
Beam/arc current & $\mathrm{I}$ & 0.35 & 5000 & $\mathrm{~A}$ \\
Beam/arc radius & $\mathrm{a}$ & 0.005 & 0.1 & $\mathrm{~m}$ \\
Pool superheat & $\Delta T$ & 200 & 200 & $\mathrm{~K}$ \\
\hline
\end{tabular}

In EBBM, $M \approx 1$, indicating that these forces are of comparable magnitude. In VAR, since $M \approx 0.01$, buoyancy forces are much greater than Marangoni forces.

We now estimate the typical value of the Lorentz force in the two processes. Assuming the current is spread evenly over a disc of radius a, we find that the average force is given by:

$$
\mathrm{F}_{\mathrm{e}}=\frac{\mu_{0} \mathrm{I}^{2}}{3 \pi^{2} \mathrm{a}^{3}}
$$

We compare this with the typical buoyancy force:

$$
F_{b}=\frac{\partial \rho}{\partial T} \Delta T g
$$

and find that $\frac{F_{e}}{F_{b}} \approx 2 \times 10^{-4}$ in EBBM, demonstrating that only buoyancy and Marangoni forces are important in determining the fluid flow. Conversely, in VAR, $\frac{F_{c}}{F_{b}} \approx 5$ and electromagnetic forces are comparable to buoyancy throughout the melt pool. Note that magnetic forces are dependent on the square of the current in the melt, so loss of half of the current would reduce these forces by a factor of four. Hence, it is important to know what proportion of the current reaches the ingot in a real installation. Evaluation of the Joule heating indicates that it is not significant in either process.

\section{VAR Mode}

\section{Validation}

To validate the macromodel it was compared to previously published work simulating the VAR process (shown schematically in Figure 1). The model was run with the values given by Jardy and Ablitzer ${ }^{6}$ in their study of fluid flow during the pilot scale VAR remelting of Zy4. They measured the pool shape experimentally (drawn as the dashed line in Figure 2) and used it as a boundary condition to determine the flow by solving the Navier-Stokes, Maxwell's, and Fourier equations. They assumed steady state and treated the problem as axisymmetric. With these conditions they calculated a maximum velocity of $0.013 \mathrm{~m} / \mathrm{s}$ when half of the melting current reaches the pool, or $\alpha=0.5$. In this study the location of the solidification front was not pre-specified, as it was by Jardy and Ablitzer, therefore values of the heat transfer coefficient, $h_{c}$, and the latent heat had to be approximated and are listed in Table II. A grid of $30 \times 100$ control volumes was used to simulate the $0.1 \times 0.5 \mathrm{~m}$ domain.

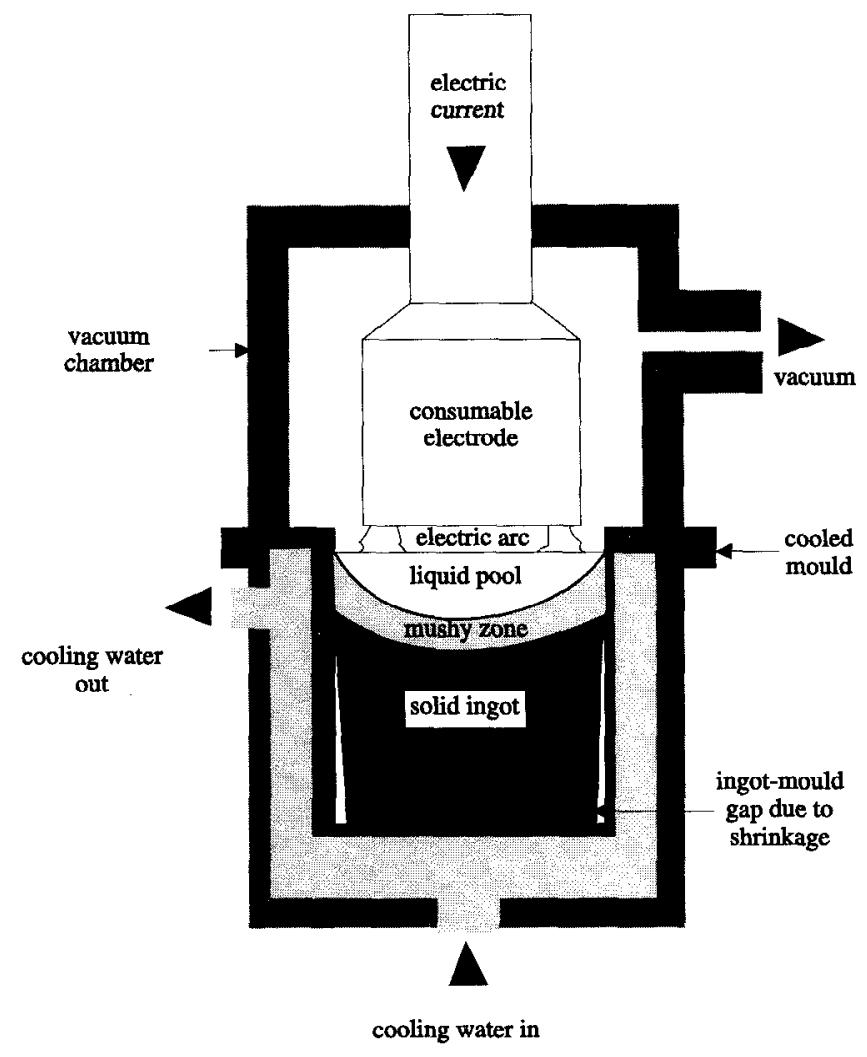

Figure 1. Schematic diagram of a VAR installation.

Table II. Approximated values used for VAR comparison simulation to Jardy and Ablitzer ${ }^{6}$ and

\begin{tabular}{|c|c|c|c|}
\hline Property & & Value & Units \\
\hline Density & $\rho$ & $6000-0.1 T$ & $\mathrm{~kg} / \mathrm{m}^{3}$ \\
\hline Ingot radius & $\mathrm{L}$ & 0.10 & $\mathrm{~m}$ \\
\hline Electrode (arc) radius & $r$ & 0.08 & $\mathrm{~m}$ \\
\hline Arc current & I & $5000 \times \alpha$ & A \\
\hline Liquidus temperature & $T_{l}$ & 2073 & $\mathrm{~K}$ \\
\hline Solidus temperature & $T_{s}$ & 2023 & $\mathrm{~K}$ \\
\hline Latent heat & $L$ & 200,000 & $\mathrm{~J} / \mathrm{kg}$ \\
\hline $\begin{array}{c}h_{c} \text { for top } 0.05 \mathrm{~m} \text { of } \\
\text { ingot/crucible contact }\end{array}$ & $h_{c}$ & $3000,\left(T_{a m b}=273 \mathrm{~K}\right)$ & $\mathrm{W} / \mathrm{m}^{2}$ \\
\hline $\begin{array}{l}h_{c} \text { for rest of ingot/crucible } \\
\text { contact }\end{array}$ & $h_{\mathrm{c}}$ & $100,\left(T_{a m b}=273 \mathrm{~K}\right)$ & $\mathrm{W} / \mathrm{m}^{2}$ \\
\hline Emissivity & $\varepsilon$ & $0.2,\left(T_{a m b}=273 \mathrm{~K}\right)$ & \\
\hline Gibbs-Thomson coefficient & $\Gamma$ & $2 \times 10^{-7}$ & $\mathrm{mK}$ \\
\hline Liquid diffusion coefficient & $D$ & $5 \times 10^{-9}$ & $\mathrm{~m}^{2} / \mathrm{s}$ \\
\hline liquidus slope & $m$ & -4 & K/wt.\% \\
\hline bulk composition & $C_{\theta}$ & 20 & wt: \% \\
\hline distribution coefficient & $k$ & 0.2 & \\
\hline
\end{tabular}
for calculating typical microstructural features.

The flow patterns and molten pool shapes predicted in the present study are shown in Figures 2(a) to 2(c) for $\alpha$ values of $0,0.5$, and 1 respectively. The maximum velocity predicted with $\alpha=0.5$ is $0.015 \mathrm{~m} / \mathrm{s}$, in excellent agreement with Jardy and Ablitzer's value of $0.013 \mathrm{~m} / \mathrm{s}$, with the difference possibly due to the pool shape being calculated rather than imposed. 


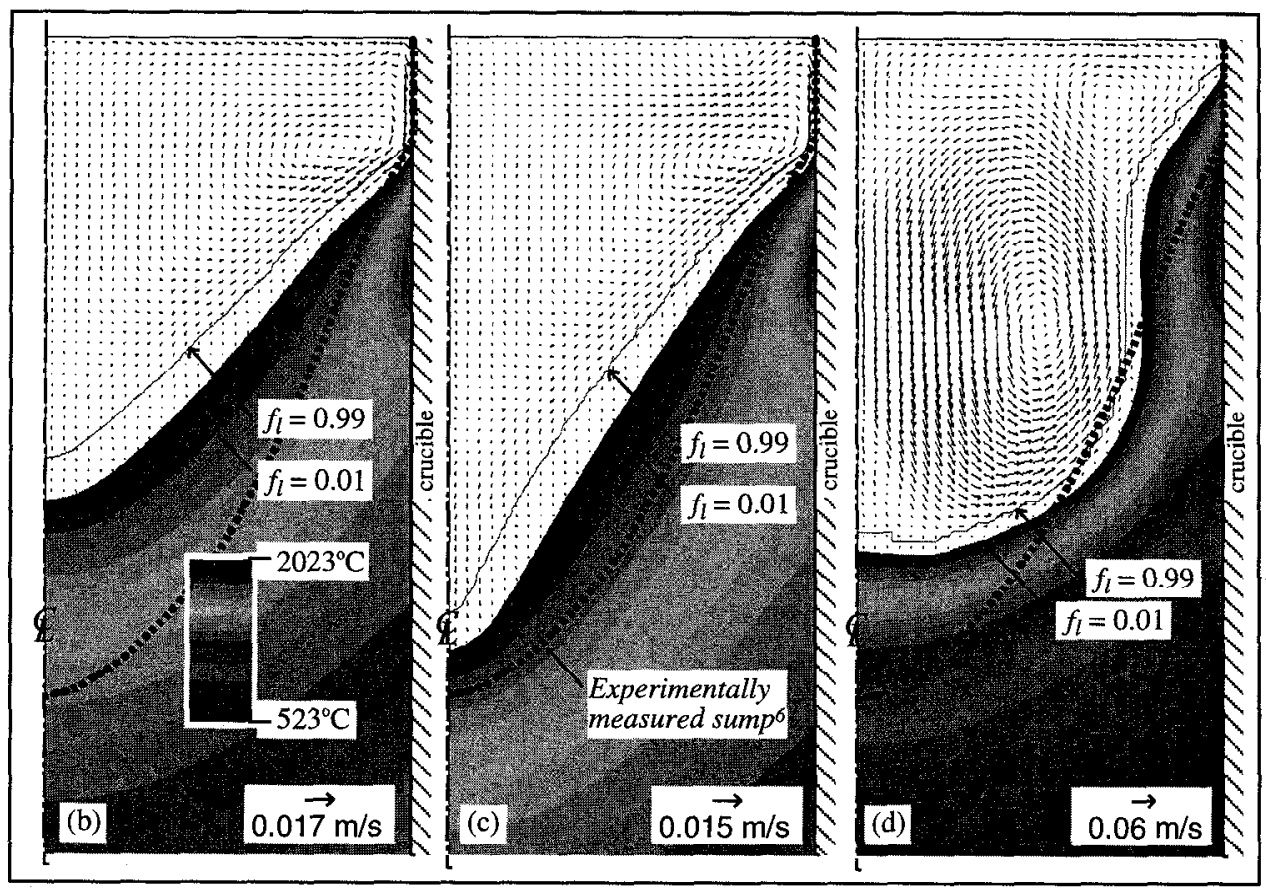

Figure 2. Calculated pool shape and velocity fields in a VAR ingot for: (a) $\alpha=0.0$; (b) $\alpha=0.5$; and (c) $\alpha=1.0$. The pool shape measured by Jardy and Ablitzer $^{6}$ is shown as the heavy dashed line.

Comparing the pool shapes calculated in the present study to that experimentally measured by Jardy and Ablitzer, the experimentally measured pool shape appears to fall between the $\alpha=0.5$ and $\alpha=1.0$ predictions (see Figures 2(b) and 2(c)). The calculated pool shape is strongly dependent on the heat transfer coefficient which was not known, hence the difference in pool depth can be attributed to any error in the approximate values used for $h_{c}$.

\section{Microstructural Predictions}

The importance of the melt pool shape on the final properties is illustrated by comparing the microstructural predictions for the two extremes of VAR with no EMF calculation (Figure 2(a)) and with the full melting current transferred (Figure 2(c)). Using the macromodel to determine $G$ and $V$ and the approximate values for the material properties as listed in Table II, the values for $\lambda_{1}$ were calculated using equation (11) and are shown in Figure 3.

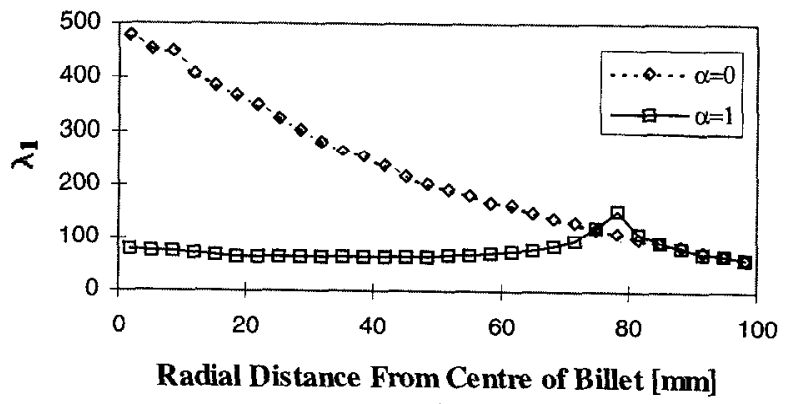

Figure 3. Predicted $\lambda_{1}$ values in a VAR ingot for $\alpha=0.0$ and $\alpha=1.0$.
When $\alpha=0$, the flow at the bottom of the sump is slow and the isotherms become stratified, with small thermal gradients, as is seen by the distance between the $f_{l}=0.01$ and $f_{l}=0.99$ contours. This leads to large $\lambda_{1}$ predictions. The strong counter-clockwise flow when $\alpha=1$ produces much higher gradients, and hence signiticantly smaller $\lambda_{1}$ predictions.

Using equation (14) the predictions for $\lambda_{2}$ are shown in Figure 4. Because the simulation was steady state, the value for $t_{s}$ was calculated by taking the distance between $T_{s}$ and $T_{l}$ parallel to the pull direction and dividing by the pull velocity. This reaches an extreme condition when the solidification front is perpendicular to the pull direction, as is the case at a radius of $78 \mathrm{~mm}$ for the $\alpha=1.0$ simulation. The primary dendrites are growing normal to the pull velocity, giving a long ripening time for the secondary arms. For most of the ingot, the EMF increases the thermal gradients,

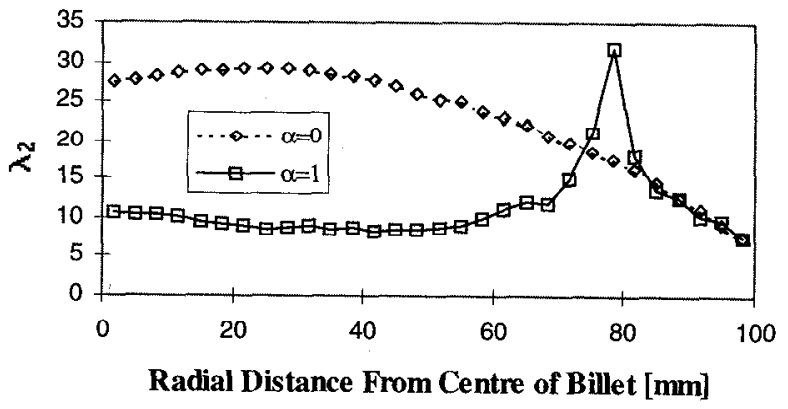

Figure 4. Predicted $\lambda_{2}$ values in a VAR ingot for $\alpha=0.0$ and $\alpha=1.0$. 
reducing the size of the mushy zone, and hence producing a finer microstructure or smaller $\lambda_{2}$ values. However, the interaction of the EMF on the pool shape produces a region of growth normal to the pull direction, giving an inversion of the general trend. Comparing of the predicted $\lambda_{2}$ values for the two cases in Figure 4 illustrates the importance of including the EMF and of calculating the pool shape rather than specifying it a priori since both have strong influences on the final ingot microstructure.

\section{Electron Beam Button Melting Model}

\section{Experimental Methods}

The forming of a button by electron beam melting into a copper cooled hemispherical crucible of $168 \mathrm{~mm}$ diameter can be summarised by the following steps:

i. crucible and electrode preheating;

ii. electrode tip shaping

iii. melting of the electrode with the molten metal dripping into the crucible and a fraction of the power being applied to the partially formed button.

iv. controlled solidification of the fully formed button.

The model results were compared to two different types of experimental runs performed at the National Physical Laboratory, Teddington, UK. The first type was designed to allow characterisation of the surface flow velocities. The second type were the standard runs designed to give a controlled solidification pattern that concentrates inclusions into a central raft for simplified cleanliness characterisation. The two types of runs differ significantly only in the final stage, iv.

As described by Quested $e t a l .{ }^{7}$, the first type of run approximates a stationary beam at the centre of the button. However, since the electron beam, even though partially defocused, distributes power over a circle with a radius less than $20 \mathrm{~mm}$, the beam was scanned circumferentially at a high frequency on the surface of the button. The centre of the beam circumscribes the centre of the button at a radius varying linearly from 0 to $20 \mathrm{~mm}$ three times over the period of $60 \mathrm{~s} . \mathrm{Al}_{2} \mathrm{O}_{3}$ particles were added to the surface and the motion of these particles was tracked using a video camera to provide rough quantitative surface flow velocities.

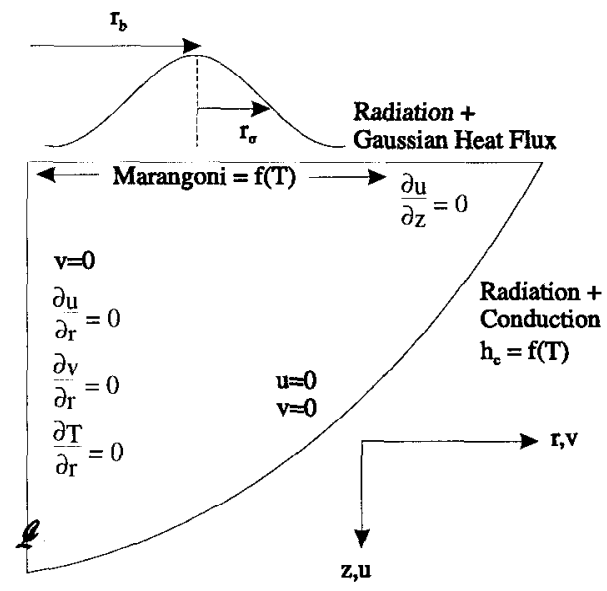

Figure 5. Schematic diagram showing the geometry and boundary conditions used to model the EBBM process.
The second type of run was a normal practice solidification cycle used to concentrate inclusions at the centre of the button, often forming a visible raft. Quested $e t$ al. ${ }^{7}$ suggested that the raft is caused by radial flow inward during stage iv, where the beam scans from the outer radius into the centre of the button. This inward radial motion is at a constant speed over a period ranging from 40 to $120 \mathrm{~s}$. Simultaneously the current is decreased linearly from full power to $0 \mathrm{~mA}$.

The buttons were examined metallographically after casting, to measure the microstructural features. The secondary dendrite arm spacing measurements, were obtained from longitudinal sections of the button which were polished and then etched in Marble's reagent (HCL $50 \mathrm{ml}$, saturated cupric sulphate solution $25 \mathrm{ml}$ and distilled water $25 \mathrm{ml}$ ). Using an SEM, the length of five consecutive secondary arms was measured and averaged to determine the spacing. The primary dendrite arm spacing, measurement technique and results are given by Ellis ${ }^{8}$.

\section{Problem Formulation}

The heat transfer and fluid flow was modelled in EBBM assuming that the flow was axisymmetric as was the distribution of heat from the electron beam. The model was solved on a grid of $40 \times 40$ control volumes for transient flow using an implicit solution with $0.5 \mathrm{~s}$ time steps. A steady state solution was obtained to use as an initial condition assuming a highly defocussed beam centred half way out the radius. The geometry and boundary conditions used are shown in Figure 5. The material properties used are listed in Table III or given in Figure 6 as a function of temperature.

Table III. Values used to simulate EBBM processing of IN718. (Note that $f(t)$ indicates the value is a function of time whilst $f(T)$ indicates a function of temperaturc.)

\begin{tabular}{c|ccc}
\hline Property & Symbol & Value & Units \\
\hline Button radius & & 37 & $\mathrm{~mm}$ \\
Button depth & & 25 & $\mathrm{~mm}$ \\
Beam current & $\mathrm{I}$ & $\mathrm{f}(t)$ & $\mathrm{mA}$ \\
Beam voltage & $\mathrm{V}$ & 25 & $\mathrm{kV}$ \\
Beam focal radius & $r_{\sigma}$ & 10 & $\mathrm{~mm}$ \\
Beam location radius & $r_{b}$ & $\mathrm{f}(t)$ & $\mathrm{mm}$ \\
Density & $\rho$ & $\mathrm{f}(T)$ & $\mathrm{kg} / \mathrm{m}^{3}$ \\
Specific heat capacity & $\mathrm{Cp}$ & $\mathrm{f}(T)$ & $\mathrm{J} / \mathrm{kg} / \mathrm{K}$ \\
Viscosity & $v$ & $5 \times 10^{-3}$ & $\mathrm{~kg} / \mathrm{m} \mathrm{s}$ \\
Surface tension gradient: & $\partial \gamma / \partial T$ & $\mathrm{f}(T)$ & $\mathrm{N} / \mathrm{m} / \mathrm{K}$ \\
Liquidus temperature & $T_{l}$ & 1609 & $\mathrm{~K}$ \\
Solidus temperature & $T_{s}$ & 1533 & $\mathrm{~K}$ \\
Latent heat & $L$ & 270,000 & $\mathrm{~J} / \mathrm{kg}$ \\
Ingot/crucible $h_{c}$ & $h_{c}$ & $\mathrm{f}\left(T_{\text {ingot }}\right)$, & $\mathrm{W} / \mathrm{m}^{2}$ \\
& & $\left(T_{a m b}=500 \mathrm{~K}\right)$ & \\
Ingot/crucible emissivity & $\varepsilon$ & 0.3, & \\
Button top emissivity & $\varepsilon_{t o p}$ & $\left(T_{a m b}=500 \mathrm{~K}\right)$ & \\
& & $\left(T_{a m b}=273 \mathrm{~K}\right)$ & \\
\hline
\end{tabular}




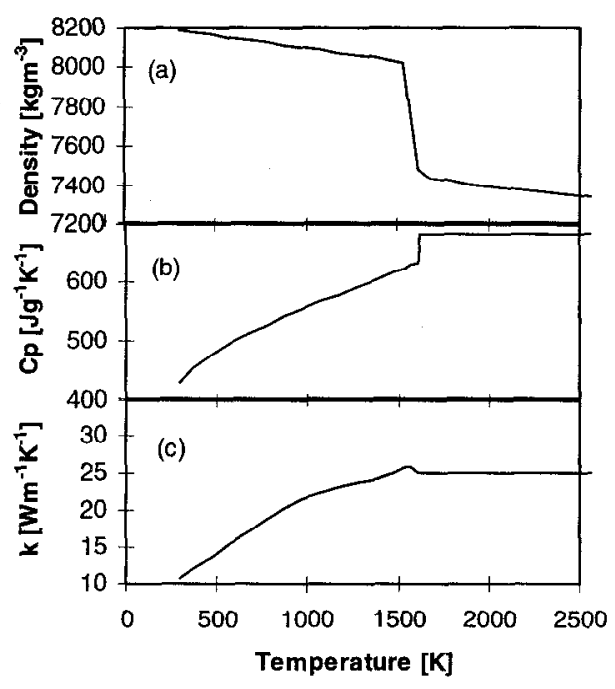

Figure 6. The temperature dependent material properties used in the EBBM simulations. (a) Density; (b) specific heat capacity; and (c) the thermal conductivity.

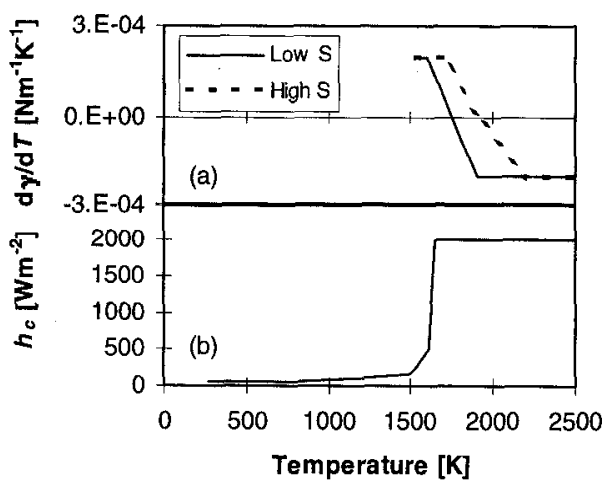

Figure 7. The temperature dependent values for (a) $\partial \gamma / \partial \mathrm{T}$ and (b) the heat transfer coefficient used in the EBBM simulations.

The values for $\partial \gamma / \partial \mathrm{T}$ were estimated using valucs provided by Mills $^{9}$ and assuming the behaviour is similar to that of sulphur in $\mathrm{Fe}-\mathrm{Ni}$ - $\mathrm{Cr}$ alloys as determined by McNallan and Debroy ${ }^{10}$. The values were included as a piece-wise linear fit. The values used are shown in Figure 7(a) for the two cases of low surfactant concentration $(6 \mathrm{ppm} \mathrm{S},<10 \mathrm{ppm} \quad 0)$, and high surfactant concentration (20 ppm S, $8 \mathrm{ppm} \mathrm{O}$ ), the CPQ and CPZ compositions of IN718 as given by Quested et al. ${ }^{7}$ respectively.

The values for the heat transfer coefficient between the ingot and mould wall, $h_{c}$, were calculated from measurements of the heat flux into a copper crucible made during the plasma remelting of IN718 into a $125 \mathrm{~mm}$ diameter cylindrical ingot. The heat flux was divided into radiative, convective, and contact components, with the $h_{c}$ value representing the contact portion.

Starting with a steady state solution, the process was modelled with the transient boundary condition of the electron beam moving across the surface providing a Gaussian distribution of heat flux, $Q$, characterised by:

$$
Q(R)=Q_{0} \mathrm{e}^{-\frac{R^{2}}{r_{\sigma}^{2}}}
$$

where $R$ is the distance from the beam centre, $r_{\sigma}$ is the beam focal radius, and $Q_{o}$ is the total flux. Given that the beam circumscribes the centre of the button at a radius of $r_{b}$, the averaged heat flux, $Q_{\theta}(r)$, can be obtained by integrating $Q$ by $\mathrm{d} \theta$, giving:

$$
Q_{\theta}(r)=\frac{1}{\pi} \int_{0}^{\pi} Q(R) \mathrm{d} \theta=Q_{o} \mathrm{e}^{-\frac{r^{2}+r_{b}^{2}}{r_{\sigma}^{2}}} \mathrm{I}_{0}\left(\frac{2 r r_{b}}{r_{\sigma}^{2}}\right),
$$

where $I_{0}$ is the modified Bessel function of the first kind and order 0 .

\section{Surface Flow Predictions}

Using the model as outlined in the previous section, two experimental runs were simulated, both with the same boundary conditions but with the low and high sulphur content being represented by the variation in $\partial \gamma / \partial T$ as a function of $T$. The electron beam motion was the same for both cases:

i. 30 seconds of $r_{b}$ varying from 25 to $5 \mathrm{~mm}$ over 2 second cycles at a power of $6 \mathrm{~kW}$;

ii. 30 seconds with no heat flux (during which time the $\mathrm{Al}_{2} \mathrm{O}_{3}$ particles were added);

iii. and finally 60 scconds of $r_{b}$ varying from 20 to $0 \mathrm{~mm}$ over 20 second cycles at a power of $6 \mathrm{~kW}$.

For the case of low $S$ the surface flow was outwards from the centre of the beam with the particles reaching a maximum velocity of approximately $0.06 \mathrm{~m} / \mathrm{s}$. The particles travelled near to the edge of the pool, but a small inward surface flow was present in the outermost region of the pool. The predicted flow pattern 78 seconds into the simulation (seconds after the pool surface had become fully molten) is shown in Figure 8(a). The predicted surface flow is outwards with a maximum value of $0.16 \mathrm{~m} / \mathrm{s}$. The predicted value is higher than the maximum velocities observed experimentally, however, the $\mathrm{Al}_{2} \mathrm{O}_{3}$ particles were not tracked in the high velocity region. As observed experimentally, a small recirculating inward flow near the edge is predicted, with a time dependent size and peak velocity. This flow is caused by the positive value of $\partial \gamma / \partial T$ at temperatures less than $1750 \mathrm{~K}$, and the size of inward flow is a function of the location of this isotherm, which is in turn dependent on the stage of remelting and the value of $r_{b}$.

For the high $\mathrm{S}$ experiments, the particles were observed to move inwards from the edge of the pool peak velocities of $0.19 \mathrm{~m} / \mathrm{s}$, moving at highest velocity shortly after leaving the edge of the pool, slowing to velocities of approximately $0.1 \mathrm{~m} / \mathrm{s}$ half way towards the centre. The particles stop before reaching the centre but on the inside of $r_{b}$. Figure $8(\mathrm{~b})$ shows the predicted flow pattern 78 seconds into the simulation. This period is when the velocities were recorded experimentally. The predicted surface flow pattern is identical to that observed experimentally, with the flow going from the outside into the centre, but turning down just before reaching the centre. The maximum velocity predicted is $0.14 \mathrm{~m} / \mathrm{s}$, lower than that observed experimentally $(0.19 \mathrm{~m} / \mathrm{s})$. 'lhis suggests that the value for $\partial \gamma / \partial T$ may be greater than that used in the simulation, or the inversion point to a negative value could be at a higher temperature.

Comparing the two cases, a reduction in the rate of change of $\partial \gamma / \partial T$ from a positive to negative value and a $150^{\circ} \mathrm{C}$ increase in the inversion point, dramatically changed the flow patterns and location of the liquidus front. The size of the mushy zone is also altered, and hence the microstructural features will be different. A comparison of Figure 8(a) to Figure 8(b) illustrates the dominance of Marangoni flow in the EBBM process. 


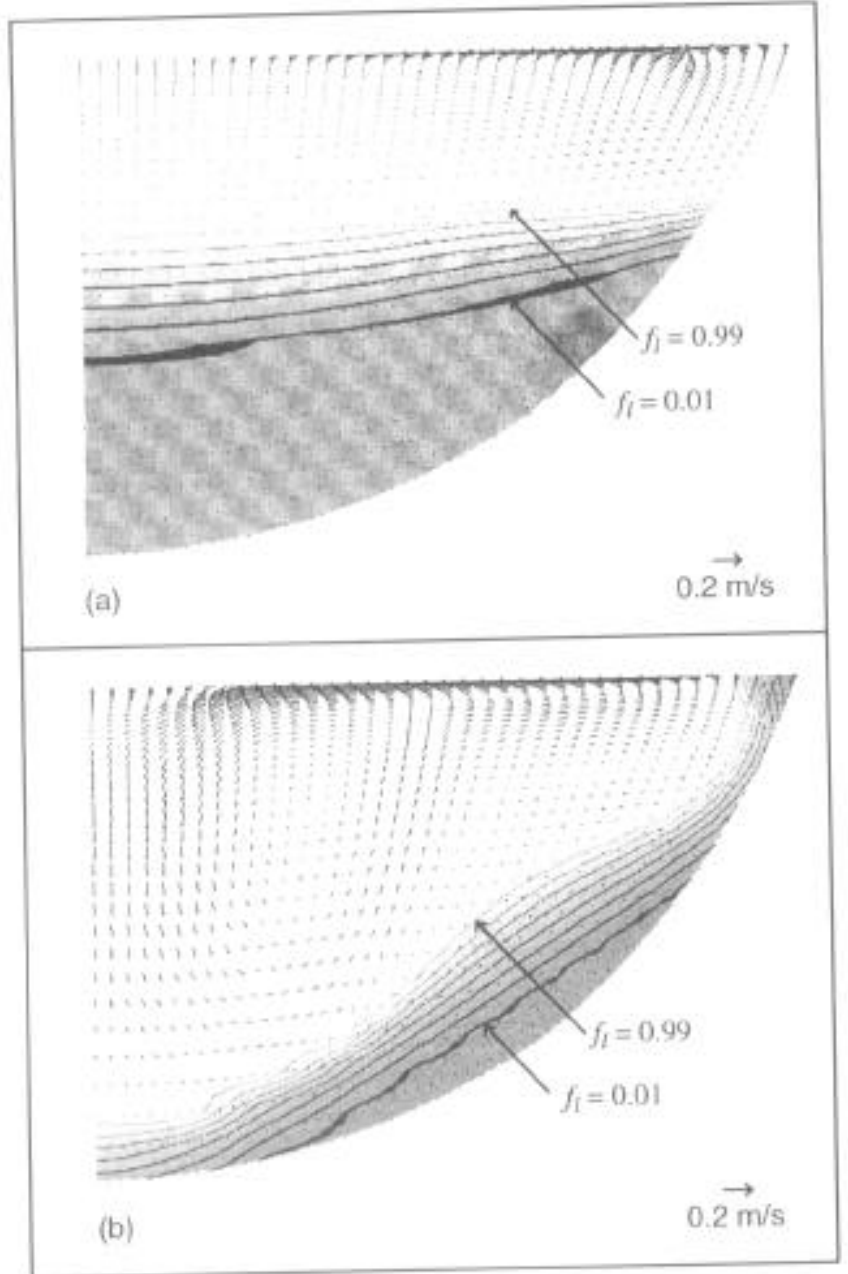

Figure 8. Predicted flow pattems using a 'central beam' with (a) a low sulphur content and (b) a high sulphur content.

\section{Microstructural Predictions}

The microstructural features of electron beam melted buttons were measured for buttons undergoing a controlled solidification stage iv processing. During this stage the electron beam moves inwards whilst the power is reduced. This process was modelled with $r_{b}$ going from $35 \mathrm{~mm}$ to zero over 120 seconds whilst the power decays linearly from $2.6 \mathrm{~kW}$ to zero. The resulting predictions for $\lambda_{t}$ are shown in Figure $9(a)$ calculated using equation (1I) with the material properties for $\Gamma, D, m, C_{m}$ and $k$ given in Table II. No predictions could be made in the bottom region of the button (cross-hatched area in Figure 9) because this area was already mushy in the steady state solution used as the initial condition for the model.

The values measured experimentally by Ellis ${ }^{k}$ are listed on the figure (in bold) beside the predicted values (in italics). Near the top of the button at the centre line the model prediction fails. This is the region to solidify last and the only area where the gradients are sufficiently low that a liquidus isotherm is predicted to enter from the top of the button due to radiative heat loss competing with conduction through the button. Experimentally this region is occasionally found to be equiaxed, indicating that the columnar dendrites can not grow in from the sides sufficientiy quickly. The
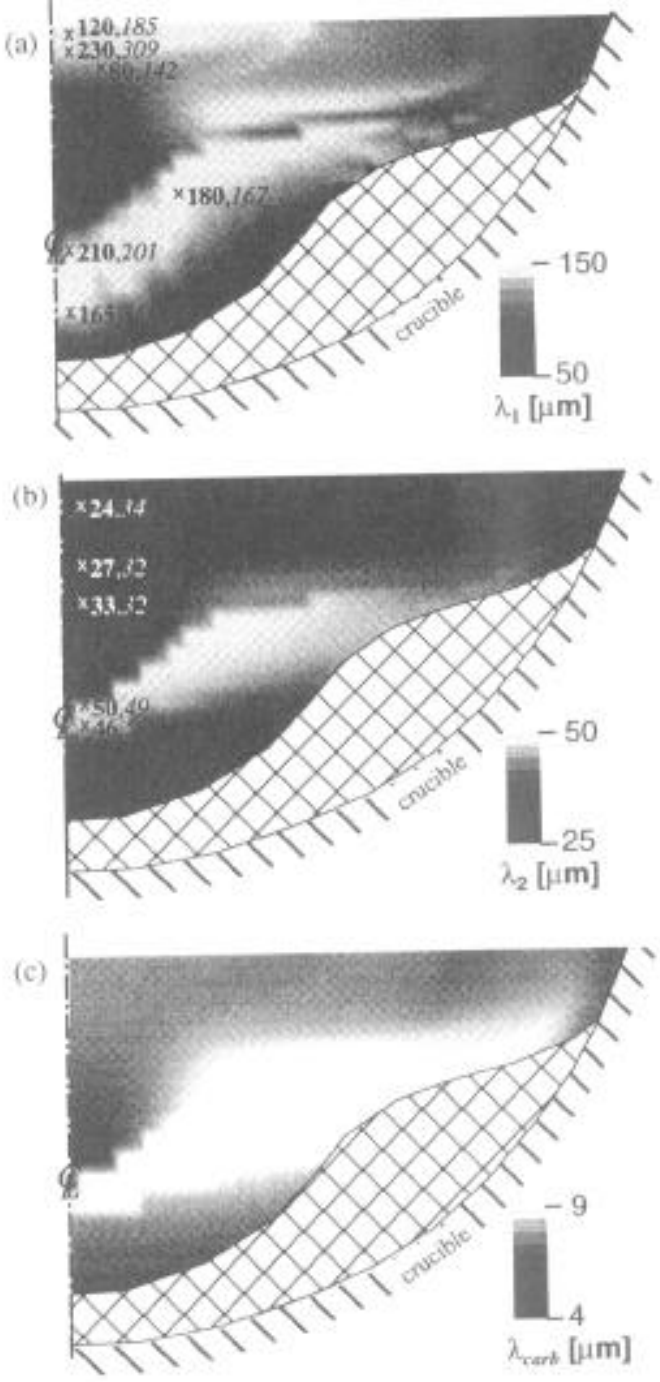

Figure 9. Predicted microstructural features for a button with a 120 s controlled solidification stage. (a) $\lambda_{1}$, (b) $\lambda_{2}$, and (c) $\lambda_{4 \omega b}$. Experimentally measured values are in bold, predicted values are in italics.

$\lambda_{1}$ model assumes that the growth is near steady state, and this assumption does not appear to hold in this region. When the columnar dendrites were found to extend to the top, the spacing was smaller than predicted, suggesting that the dendrites could not adjust their spacing in this relatively smail distance. Pratt and Grugel ${ }^{\mathrm{It}}$ have shown experimentally that the $\lambda_{1}$ adjust to order of magnitude changes in pull velocity during directional solidification experiments, suggesting either a slow response time to changes in thermal conditions or that the stable growth regime is large, adding a hysteresis effect.

The predictions for $\lambda_{2}$ are shown in Figure $9(\mathrm{~b})$ calculated using equation (14). The correlation of predicted values to experimental is good. Predicted values for $\lambda_{\text {rarb }}$ are shown in Figure $9(\mathrm{c})$ calculated using equation (15). The carbide spacing was not measured experimentally. 


\section{Conclusions}

Either the Lorcntz or Marangoni force can be the governing factor for flow during secondary remelting processes, depending on the length scale and thermal gradients. The relative importance of these forces can be estimated by dimensionless analysis. A macromodel of the fluid flow and heat transfer was coupled to microstructural models to show that the changes in flow, caused by the different driving forces, have a large impact on the final microstructural features of the superalloy.

Many microstructural predictions can be made with straight forward correlation, for example $\lambda_{1}, \lambda_{2}$, and $\lambda_{\text {carb }}$. However, in the case of $\lambda_{1}$ a more complex model may be required to account for the response time and hysteresis of $\lambda_{1}$ to changes in thermal conditions.

The comparison of predicted to observed surface flows in electron beam melted buttons confirms that the Marangoni force is the main driving force for fluid flow in the EBBM process. Minor variations in the dependency of the surface tension on temperature can cause dramatically different flows, as shown by increasing the concentration of the surfactant sulphur from 6 to $20 \mathrm{ppm}$.

\section{Acknowledgements}

The authors would like to thank: Peter Quested, David Hayes and Ken Mills at the National Physical Laboratory for their assistance with both the experiments and the provision of material property data; the IRC in Materials for High Performance Applications, Birmingham, for providing experimental measurements of superalloy ingot to crucible heat fluxes; and Inco Alloys Ltd. for supplying both material and information. PDL would like to acknowledge the financial support of the EPSRC. RML would like to acknowledge the financial support of the DRA, Farnborough.

\section{References}

1. C. Hirsch, Numerical Computation of Internal and External Flows, Vol. 1 (John Wiley \& Sons 1988).

2. M. McLean, Directionally Solidified Materials for High Temperature Service, (The Materials Society 1983), 28-33.

3. S.-Z. Lu, J.D. Hunt, “A Numerical Analysis of Dendritic and Cellular Array Growth: the Spacing Adjustment Mechanisms", J. Crystal Growth 123 (1992), 17-34.

4. J.D. Hunt and S.-Z. Lu, "Numerical Modelling of Cellular/Dendritic Array Growth: Spacing and Undercooling Predictions", Modelling of Casting, Welding and Advanced Solidification Processes VII, Ed. M. Cross and J. Campbell, (TMS 1995), 525-532.

5. A. Jardy, D. Ablitzer, J.F. Wadier, "Magnetohydrodynamic and thermal behavior of electroslag remelting slags." Met. Trans. 22B (1991), 111-120.

6. A. Jardy and D. Ablitzer, "On Convective and Turbulent Heat Transfer in VAR Ingot Pools", Modelling of Casting, Welding and Advanced Solidification Processes V, Ed. M. Rappaz et al., (TMS 1990) 699-706.

7. P.N. Quested, D.M. Hayes, K.C. Mills, "Factors affecting raft formation in electron beam buttons", Mat. Sci. and Eng., A173 (1993), 371-377.

8. J. D. Ellis, "Quality Assurance by Electron Beam Button Melting", (Ph.D. thesis, Imperial College of Sci., Tcch. and Med., 1992).
9. K.C. Mills, private communication with author, National Physical Laboratory, Teddington, 16 October, 1995.

10. M.J. McNallan and T. Debroy, "Effect of Temperature and Composition on Surface Tension in $\mathrm{Fe}-\mathrm{Ni}-\mathrm{Cr}$ Alloys Containing Sulfur", Met. Trans. 22B (1991), 557-560.

11. R.A. Pratt, R.N. Grugel, "Microstructural Response to Controlled Accelerations During the Directional Solidification of Al-6 wt.\% Si Alloys", Materials Charact. 31 (1993), 225-231. 